\title{
Clarice Lispector, Nathalie Sarraute oder die literarische Behandlung autobiographischer Oberflächen
}

Während des nun ins Auge gefassten Zeitraums erhielten autobiographische und autofiktionale Schreibformen eine solche Bedeutung, dass man ohne jede Übertreibung sagen könnte, dass autobiographisches Schreiben in seinen vielfältigen Variationen zu einer der Hauptgattungen der Literaturen im Zeichen der Postmoderne geworden ist. Diese Aussage hat das Zeug, zumindest all jenen als paradox zu erscheinen, die unserer Vorlesung aufmerksam gefolgt sind. Denn dann wissen Sie, dass sich gerade in den sechziger Jahren unter dem Druck des Textualitäts-Dogmas im Umfeld der Gruppe Tel Quel die Positionen derart radikalisierten, dass immer schärfere Angriffe gegen eine im Abendland traditionell vorherrschende Subjektphilosophie und eine im literarischen Bereich immer vehementere Hinterfragung der Begriffe „Subjekt“ oder „Autor“ erfolgten. Dies subvertierte die Grundlagen literarischen Schreibens selbst - wie am Beispiel von Michel Butors Mobile gezeigt - und ließ nach neuen textuellen Ufern Ausschau halten.

Dabei ging es schon bald nicht mehr um Subjektivität, insofern das Subjekt gleichsam abgeschafft worden war, zusammen mit allem Muff und den Konventionen der Psychologie und des psychologischen Romans. Die ,Tiefe‘ literarischer Figuren erschien als nicht weniger bürgerlich, rückständig und reaktionär als die herkömmliche Darstellung literarischer Subjektivität, wie sie sich kanonisch innerhalb von Erzählwerken in einer Figurenkonstellation ausdrückt. Nicht die Figuren und deren Variationen des Autors, sondern allerhöchstens die Autorfunktion und deren Textualität waren Themen, denen man sich (zumindest in den Theorie-Eliten) noch mit großem Eifer zuwandte.

Die Figur des Lesers rückte in den Mittelpunkt und die Markierung des Autors schien in den ausgehenden sechziger sowie beginnenden siebziger Jahren keine größere Aufmerksamkeit mehr zu verdienen. Und doch: Gerade jener Theoretiker und Essayist, der durchaus öffentlichkeitswirksam den Tod des Autors verkündet hatte, legte im Jahre 1975, also gerade einmal sieben Jahre nach seinem vielbeachteten Aufsatz, selbst eine Autobiographie vor, zudem in jener Reihe „Ecrivains de toujours“, in der er zu Beginn seiner Karriere eine dem Historiker Michelet gewidmete Biographie veröffentlicht hatte. War Barthes damit definitiv zu einem Schriftsteller geworden?

Roland Barthes par Roland Barthes war durchaus keine gewöhnliche Autobiographie. Uns fehlt ein wenig die Zeit, uns mit diesem für die Entwicklung 


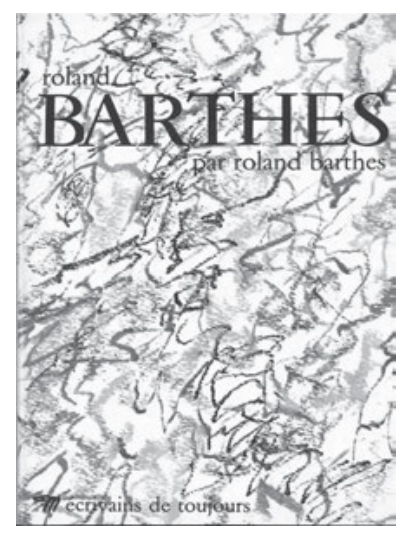

Abb. 108: Cover von Roland Barthes par Roland Barthes, 1975.

der Gattung nicht unwichtigen Band zu beschäftigen. ${ }^{1}$ Doch sei an dieser Stelle zumindest festgehalten, dass bereits der Titel umschrieb, dass es sich um zwei mit diesem Namen ausgestattete Erzählinstanzen handelte, wobei der zweite Roland Barthes die erste Instanz in der dritten Person ansprach und in der für Barthes charakteristischen Kurzschreibweise konturierte. Die für eine herkömmliche Autobiographie fundamentale Spaltung zwischen erzählendem Ich und erzähltem Ich fand sich so in anderer Spiegelung wieder, auch wenn kein Zweifel daran bestehen konnte, dass sich autobiographische Schreibformen auch in der dritten Person um jene textexterne Person drehten, die zudem in Photographien im Band abgebildet war und den französischen Schriftsteller zeigte.

Zudem handelte es sich bei diesem Text nicht etwa um die kontinuierliche Entwicklung einer Person und Persönlichkeit, mit psychologischer Tiefe und in historischer Entfaltung, sondern um eine alphabetisch angeordnete Abfolge kurzer Fragmente, die sich bestimmten Themenstellungen zuwandten und mit einem gewissen Erfolg versuchten, sich gegen jegliche Anordnung zu einer kontinuierlichen Entwicklung zu sperren. All dies hielt Barthes freilich nicht davon $\mathrm{ab}$, auf ironische und selbstironische Weise einen Plan seiner eigenen phasenhaften Entwicklung in den Text zu integrieren, gleichsam das Simulacrum seiner eigenen theoretischen und textuellen Praxis aufzustellen.

So kehrte in Roland Barthes par Roland Barthes das Subjekt nicht einfach in Gestalt des sich selbst behandelnden und darstellenden Autors zurück, sondern als Abfolge diskontinuierlicher Markierungen. Es handelt sich dabei um eine zwischen Fiktionalem und Diktionalem oszillierende Konstruktion, die hochgra-

1 Verwiesen sei auf die speziell den autobiographischen Schreibformen des Lebens gewidmete Monographie von Ette, Ottmar: LebensZeichen. Roland Barthes zur Einführung. Hamburg: Junius Verlag 2011. 
dig ambivalent blieb, als bewusste Konstruktion markiert war, nur in geringem Maße eine psychologische Konsistenz besaß, gleichwohl aber jenem Verlangen des Publikums ein Stück weit entgegenkam, das Philippe Lejeune im selben Jahr mit der Metapher des „pacte autobiographique“ umschrieb. ${ }^{2}$ Denn trotz aller schreibpraktischen Verfahren und Mechanismen, trotz der Tatsache, dass hier das Subjekt nicht einfach wieder in den Text zurückkehrte, sondern sich als Subjektfiktion präsentierte, hielt das Lesepublikum am autobiographischen Pakt fest, identifizierte trotz aller Verfremdungseffekte das textuelle Gebilde „Roland Barthes“ mit jener textexternen Realität, jenem realen Autor, der den Namen Roland Barthes trug.

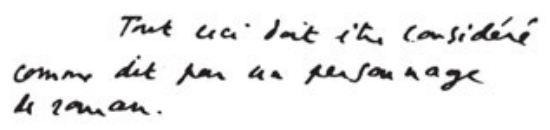

Abb. 109: Roland Barthes' Handschrift, aus Roland Barthes par Roland Barthes, 1975.

Eine weitere wichtige Dimension, die uns freilich schon mehrfach beschäftigte, ist die Dimension der Spiegelung, welche wir schon von Maurice Blanchot in Le livre à venir adressiert fanden. Die autobiographische Spiegelung - und wir werden noch Beispiele dafür kennenlernen - lässt sich als eine Dimension von Autoreflexivität verstehen, die den Roman im Zeichen der Postmoderne zusammen mit einer metaliterarischen Diskursivität auszeichnet. Diese Kombination oder auch Engführung von metaliterarischem Diskurs und auto- oder metabiographischem Diskurs findet sich in der Tat zunehmend seit den siebziger Jahren im Gefolge der erwähnten Theoriebildungen; und so zeigt sich, dass durch die Hintertür der Theorie gleichsam das Verlangen, autobiographische Texte zu schreiben, metaliterarisch wieder die Bühne zu betreten begann. Diese literarästhetische Erfahrung war zwar paradox, passte aber eben deshalb recht gut in ein postmodernes Denkschema.

Die Abweichungen vom traditionellen Modell der modernen Autobiographie waren dabei so eklatant, dass eine ständige Überschreitung ihrer Grenzen in jenen Jahren literarisch erprobt und durchgeführt wurde, was freilich verbunden mit dem ständigen experimentellen Impetus dem autobiographischen Schreiben einen zunehmend expandierenden und immer breiteren Raum einnehmenden Status im Spiel der praktizierten Gattungen verschaffte. Auf diese Weise wurden die variantenreichen Formen autobiographischen und autofiktionalen Schreibens, wie wir diesen Bereich etwas vorsichtiger nennen könnten, zu einer recht

2 Vgl. Lejeune, Philippe: Le Pacte autobiographique. Paris: Seuil 1975. 
vorherrschenden und weithin sichtbaren literarischen Form der Literaturen im Zeichen der Postmoderne. Man schrieb wieder über sich.

Aber mit Kautelen: Was von sich behauptet, nicht einfach Ich zu sein, kann umso leichter gespiegelt werden! Im Schutze von Theorie und metaliterarischem Diskurses wurde autobiographisches Schreiben seit dem Auslaufen des Höhepunkts der Theoriedebatte in Frankreich ungeheuer produktiv, zumal es auf das erhöhte Interesse geneigter Leser und Leserinnen sowie entsprechend interessierter Verlage rechnen konnte. Denn die Autobiographie rechnete sich auch für letztere, nicht zuletzt auch bei Theoretikern und großen Experimentatoren des Romans, denken wir dabei an Roland Barthes oder Alain Robbe-Grillet, Nathalie Sarraute oder Julia Kristeva, Gayatri Chakravorty Spivak oder Umberto Eco.

Autobiographische Schreibformen waren gewiss zu Zeiten der großen Theoriedebatten in Frankreich nicht gänzlich von der Bildfläche verschwunden. Und doch hing ihnen der Geruch reaktionärer bürgerlicher Selbstbespiegelung, arroganten Festhaltens an alten, überkommenen Konventionen an. Erinnern wir uns aber daran, was Umberto Eco scherzhaft, aber zutreffend über die Postmoderne sagte, so dürfen wir uns autobiographisches Schreiben ,nach' der Theorie im Epizentrum Frankreich als hintergründiges Spiel vorstellen. Seit die psychologische Tiefe abgeschafft wurde, die Oberflächen einer funktionalen Sichtweise von Figuren erkennbar wurden, seit auch die Figur des Ichs zu einer grammatikalischen Figur von Autoreflexivität und nicht von Offenbarung geworden war, konnten sich autobiographische und autofiktionale Schreibformen im Zeichen der Postmoderne wieder neu beleben - ohne jedes schlechte Gewissen.

Ich möchte mich im Folgenden mit zwei Texten von Autorinnen beschäftigen, die - so scheint mir - aus unterschiedlichen Gründen für unsere Fragestellung einen wichtigen Beitrag geleistet haben. Dabei handelt es sich in beiden Fällen keineswegs um Autobiographien im eigentlichen, traditionellen Sinne. Das Buch der brasilianischen Autorin Clarice Lispector wird im Untertitel als Roman bezeichnet, und es würde uns in der Tat sehr schwer fallen, diesen Roman als Autobiographie misszuverstehen. Und doch trägt dieser feinsinnige literarische Text - wie wir noch sehen werden - wichtige Elemente bei zu jenen Formen autobiographischen Schreibens, die uns vordringlich interessieren. Das Buch von Nathalie Sarraute, das den fast schon provozierenden Titel Enfance trägt, entwickelt nicht weniger interessante Formen autoreflexiven Schreibens, die ein Jahrzehnt später, während der achtziger Jahre, in neuen Formen möglich geworden waren. Doch wenden wir uns zunächst der Brasilianerin Lispector zu, denn ihr gleich zu besprechendes Buch führt uns zurück in den experimentellen Raum der ersten Hälfte der siebziger Jahre und zugleich zu einer Grundfrage weiblichen Schreibens: Wie ist Geschlechterdifferenz in einen Text integrierbar und wie ist sie literarisch vermittelbar? 
Vergewissern wir uns zunächst einiger für uns interessanter Biographeme aus dem Leben der in Europa stets als Geheimtipp gehandelten brasilianischen Autorin! Clarice Lispector wurde am 10. Dezember 1920 im ukrainischen Tschetschelnik geboren und starb am 9. Dezember 1977 in Rio de Janeiro. Sie stammte aus einer jüdischen Familie, trug den hebräischen Namen Chaya (gleich „Leben“) und kam im Alter von zwei Monaten nach Brasilien, wo sich die Familie im armen Nordosten des Landes niederließ. Bis zu ihrem neunten Lebensjahr lebte Clarice Lispector in der Regionalhauptstadt Recife. Die Namen aller Familienmitglieder ihre Eltern sprachen nur Jiddisch - wurden nach der Übersiedelung brasilianisiert.

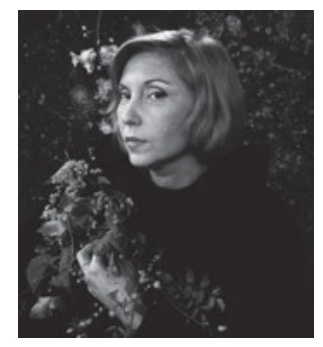

Abb. 110: Clarice Lispector (Tschetschelnyk, Ukrainische Sozialistische Sowjetrepublik, 1920 - Rio de Janeiro, 1977).

Nach dem Tod der Mutter zog sie 1934 mit dem Vater und ihren beiden Schwestern nach Rio de Janeiro, wo sie das Gymnasium besuchte und 1937 ein Jurastudium aufnahm. Nach diesem arbeitete sie zunächst als Lehrerin und Journalistin für verschiedene Zeitungen, wobei sie wohl die erste in der Familie war, die dem Jiddischen perfekt eine zweite Sprache hinzufügte. Früh schon wollte sie Schriftstellerin werden; und bald erschienen ihre ersten Erzählungen in Zeitungen und Zeitschriften.

Gegen familiäre Vorbehalte heiratete sie 1943 einen katholischen Studienkollegen und angehenden Diplomaten. Sie schrieb an ihrem ersten Roman, Perto do coração selvagem (Nahe dem wilden Herzen), der bei seiner Veröffentlichung 1944 mit großer Begeisterung aufgenommen und ausgezeichnet wurde. Ihrem Gatten folgte sie im diplomatischen Dienst und lebte von 1945 bis 1949 in Neapel sowie der Schweiz und von 1952 bis 1959 in Washington, wo ihre beiden Söhne auf die Welt kamen. Nach der Scheidung 1959 ließ sie sich mit ihnen in Rio de Janeiro nieder und arbeitete als Journalistin und Übersetzerin für große brasilianische Tageszeitungen und Zeitschriften. Neben ihren journalistischen Kolumnen folgten in den fünfziger Jahren in rascher Folge Romane und Erzählungen, die ihren literarischen Ruhm festigten. In den sechziger und siebziger Jahren entstanden neben weiteren Romanen und Erzählungen, die ins Englische, Französische, Deutsche, Tschechische und Spanische übersetzt wurden, zahlreiche Essays, 
Kinderbücher, Chroniken, Drehbücher, Reportagen und kurze Erzählprosa. Zu ihren größten Erfolgen zählt der vom umstrittenen Star-Übersetzer Curt MeyerClason ins Deutsche übertragene Roman A maçã no escuro (Der Apfel im Dunkeln). Aber auch andere Erzählwerke der als schwer verständlich geltenden und oftmals depressiven Schriftstellerin stießen bei der internationalen Kritik auf sehr positive Resonanz.

Als sie in ihrer Wohnung im 13. Stock in Rio de Janeiro versehentlich einen Hausbrand auslöste und verzweifelt Manuskripte und Bücher zu retten versuchte, erlitt sie starke Verbrennungen und konnte ihre rechte Hand nur noch unter Schmerzen gebrauchen. 1968 nahm die mit zahlreichen Preisen ausgezeichnete Autorin an den Demonstrationen gegen die damalige brasilianische Militärdiktatur teil. Nach ihrem Tod an einem Krebsleiden einen Tag vor ihrem 57. Geburtstag wurde sie auf dem jüdischen Friedhof von Rio de Janeiro beigesetzt.

Bei dem 1969 erstmals publizierten Roman Uma aprendizagem ou O livro dos prazeres haben wir es mit einem Text der ausgehenden 60er Jahren zu tun, geschrieben von einer Autorin, die unter anderem durch ihre langjährigen Auslandsaufenthalte sehr vertraut war mit verschiedensten kulturellen Kontexten. Aufgrund ihrer jüdisch-ukrainischen Herkunft, aber auch ihres für Brasilien leicht fremdländischen Aussehens erschien sie, die in Europa überall als Europäerin durchgegangen wäre, in ihrer brasilianischen Heimat als ,die Fremde‘, ein Bild, das ihr bis zum Lebensende anhing.

Der Titel des in der Folge zu besprechenden Bandes, Eine Lehre oder Das Buch der Lüste spielt auf die Tradition erotischer Literatur an, was der Text in gewisser Weise einlöst, kommt es doch gegen Ende des Romans zu einer Verschmelzung beider Protagonisten im wiederholten Liebesakt, der ein ums andere Mal zuvor aufgeschoben worden war. Doch ist diese Lehre, dieser im Titel angekündigte Lernprozess, nicht etwa dem erotischen Erleben gewidmet, sondern viel fundamentaler der Beziehung zwischen Mann und Frau. Dabei ist die Frau diejenige, aus deren Perspektive und Blickwinkel das Spiel der Geschlechterdifferenz beleuchtet wird. Eine Autobiographie ist dieser Text dabei keineswegs, wohl aber besitzt die weibliche Figur einige Biographeme, welche sich autofiktional mit der textexternen Autorin Clarice Lispector in Beziehung setzen lassen. Versuchen wir, uns mit den Grundstrukturen dieses Romans vertraut zu machen!

Dem Lesepublikum mag zunächst ins Auge fallen, dass es sich um einen fiktionalen Text handelt, der keineswegs Breite und Totalität darzustellender Welt in sich aufgenommen hat. Wohl aber enthält er ein breites Spektrum an möglichen Reaktionsweisen, welche eine Frau im Kontakt mit einem Mann auf diesen Buchseiten vorzuführen scheint. Die gesamte Handlung - wenn man denn überhaupt von Handlung im eigentlichen Sinne sprechen kann - wird von kleinen Bewegungen bestimmt, die wir mit Nathalie Sarraute als „Tropismes“, als kleine, 
unmerkliche Bewegungen, beschreiben können. Diese bestimmen in der Folge das gesamte Entwicklungsgerüst des Erzählens.

Denn der Roman besitzt - wie schon angedeutet wurde - durchaus eine romaneske Entwicklung. Geschult an der Beobachtungsgenauigkeit einer Virginia Woolf, deren Schreiben die brasilianische Autorin stets erfreute, zeigt Uma aprendizagem ou $O$ livro dos prazeres die Entwicklung der Liebesbeziehung zwischen einer wie Clarice Lispector selbst aus Pernambuco stammenden Grundschullehrerin und einem an der Universität arbeitenden Philosophieprofessor, was auf struktureller Ebene beide in eine Beziehung gleichzeitig der Analogie und der Differenz bringt. Denn beide geben ihr Wissen an zu ihren Veranstaltungen kommende jüngere Menschen weiter, doch tun sie dies auf unterschiedlichen Niveaus.

Diese strukturelle Anlage führt erwartungsgemäß eine Hierarchie zwischen beiden bezüglich der Ebene ihres Wissens und mehr noch des Diskurses ein. Denn im Gegensatz zu Lori, die eigentlich auf den Namen Loreley getauft wurde, befindet sich Ulysses, der als suchender Odysseus einen nicht weniger bedeutsamen Namen trägt, im Besitz des Wissens-Diskurses und ist daran gewohnt, nicht nur über das Wissen selbst, sondern auch über dessen vollumfängliche Verwendung zu verfügen. Der Hochschullehrer ist jederzeit in der Lage, die jeweilige Situation seiner Partnerin zu durchschauen; er gibt ihr Ratschläge, bisweilen geradezu Befehle, da er sich im Diskurs der Erkenntnis und im Vollbesitz der Erfahrung weiß. Nicht umsonst unterrichtet er Philosophie, ist also professioneller Freund der Sophia, der Wahrheit, und daran ausgerichtet, Wissen über die Welt zu einem Weltbild zu formen.

Gegen diesen männlichen (und letztlich phallogozentrischen) Diskurs brandet immer wieder die eigene Logik des weiblichen Diskurses an, der sich im Gegensatz zu ersterem nicht zu einem strukturierten und durchdachten System zu konfigurieren scheint. Es handelt sich im Grunde um ein zugleich gebrochenes, sich immer wieder um sich selbst und seine eigenen Brechungen bemühtes, bisweilen aber auch den Diskurs des Wissens ironisch unterlaufendes Sprechen, welches von einer radikalen Alterität zwischen den Geschlechtern ausgeht. Die in diesem fiktionalen Text vorgeführte Geschlechterdifferenz erscheint als statisch; und dennoch bringt sie eine Entwicklung zwischen diesen beiden Polen hervor.

Bei dieser Romananlage handelt es sich literar- und gattungsästhetisch gesprochen um eine ironische, bisweilen parodistische Verfremdung von Grundstrukturen des Entwicklungsromans und des Melodrams, des Liebes- und Bildungsromans der Herzen. Sie bildet eine wahre Education sentimentale, eine Bildungsgeschichte, welche beide Partner miteinander verbindet und letztlich vereint. Dabei ist sowohl die vorherige wie die spätere Entwicklung durchaus 
offen. Denn der Roman fängt ein wenig abrupt in Kleinschreibung an, wobei das Vorleben später immer wieder in Rückblicken und Flashbacks eingeblendet wird; und er hört ebenso abrupt auf mit einem Doppelpunkt, sagt Odysseus oder - wie es am Ende heißt - „der Mann“ doch „das Folgende“, was dann freilich nicht mehr abgedruckt wird. So bleibt am Ende, um mit Wolfgang Iser zu sprechen, eine Unbestimmtheitsstelle, mithin eine letzte große Leerstelle, in der sich die Lehre vielleicht in einen unendlich weiterlaufenden Prozess einer Lehre mit „h“, vielleicht aber auch eine Leere mit doppeltem „e“ ausgestaltet und auswächst. Aber dies sind Spiele mit dem übersetzten deutschsprachigen Titel, denen wir nicht zu sehr frönen sollten.

Dem Buch geht eine mit „C.L.“ signierte „Nota“ voraus, die für unsere an den Band herangetragene Frage nach dem autobiographischen beziehungsweise autofiktionalen Schreiben nicht ohne Belang ist. Ich möchte Ihnen gerne einen kurzen Auszug dieser „Nota“ präsentieren:

Dieses Buch verlangte eine größere Freiheit, als ich zu geben Furcht hatte. Es steht weit über mir. Demütig habe ich es zu schreiben gesucht. Ich bin stärker als ich. ${ }^{3}$

Was wir in dieser kurzen Passage beobachten können, ist das Auseinandertreten zwischen dem Anspruch des Buchs und den Möglichkeiten ästhetischer Einlösung, zwischen Freiheit und Angst, der Furcht vor ihr und dem literarischen Experiment. Gleichzeitig tut sich im und über das Medium des Schreibens eine Differenz auf zwischen zwei hier explizit genannten Ichs, so dass letztlich die zunächst aufgerissene Kluft durch das Schreiben transformiert und überführt wird in eine Differenz zwischen zwei Ich-Formen, von denen das eine stärker ist als das andere. Die Anklänge an Arthur Rimbauds „Je est un autre“ sind offenkundig. Vor allem aber dokumentiert sich ein Auseinandertreten zwischen zwei Ich-Figurationen, das uns im weiteren Fortgang unserer kurzen Analyse noch beschäftigen wird.

Gleich zu Beginn des Romans finden wir eine weibliche Figur vor, die im Übrigen ein Dienstmädchen beschäftigt, welche in Reflexionen darüber begriffen ist, welches Kleid sie anziehen solle, um sich besonders attraktiv zu machen. In der Folge wird der Blick in den Spiegel immer wieder die Spiegelungen des Ich begründen - ein bereits im Roman des 19. Jahrhunderts geläufiges Verfahren, punktiert dieser Blick - wie etwa in Gustave Flauberts Madame Bovary - doch die schrittweise Selbsterkenntnis der weiblichen Figuren.

3 Lispector, Clarice: Uma aprendizagem ou O livro dos prazeres: romance. Rio de Janeiro: Francisco Alves 1993, S. 13. 
Das Ich der Frau ist in die dritte Person gewendet: Es gibt also eine Erzählerstimme, die jedoch deutlich näher zur weiblichen als zur männlichen Figur steht. So könnten wir bereits auf dieser Ebene eine grammatikalische Verankerung der beiden zuvor auseinandergetretenen Ich-Figurationen vermuten. Bereits auf der ersten Seite des Romans aber tritt eine männliche Figur hinzu, jener Odysseus, der mit seinen Lehren nicht gerade hinterm Berg hält. Diese Passagen sind einem fortgesetzten inneren Monolog in der dritten Person Singular entnommen; schon der erste Satz des Textes war ja - wie ich Ihnen sagte gleichsam aus dem Nichts gekommen. So entsteht ein Romananfang, ein Incipit, ohne wirklichen Anfang:

nun Gott sei Dank, dass sie Ferien hatte, war sie zum Kleiderschrank gegangen, um sich $\mathrm{zu}$ entscheiden, welches Kleid sie anziehen sollte, um sich extrem attraktiv für das Treffen mit Odysseus zu machen, der ihr bereits gesagt hatte, dass sie beim Anziehen keinen guten Geschmack habe, sie erinnerte sich, dass er, weil Samstag war, mehr Zeit haben würde, weil er an diesem Tag die Ferienstunden an der Universität nicht gab, sie dachte daran, dass er sich allmählich für sie verändern würde, dass er zu wünschen schien, dass sie wisse, nahm an, dass er sie nur lehren wolle, ohne Schmerzen zu leben, hatte er doch einmal gesagt, dass er wolle, dass sie, wenn man sie nach ihrem Namen frage, nicht antworte „Lóri“, sondern dass sie antworten könnte „mein Name ist ich“, denn dein Name, hatte er gesagt, ist ein Ich, sie fragte sich, ob das schwarz-weiße Kleid ginge,

dann kam, direkt aus dem Bauch, wie ein fernes Schaudern der Erde, von dem man kaum wusste, ob es das Signal des Erdbebens, des Uterus, des zusammengezogenen Herzens sei, die gigantische Erschütterung eines starken aufgeschüttelten Schmerzes, der Aufruhr des ganzen Körpers - und in verschleierten Verzerrungen des Gesichts und dann des Körpers mit der Schwierigkeit eines Petroleum, das die Erde aufreißt - kam schließlich das große trockene Schluchzen, ein stummes Schluchzen ohne jeden Ton, sogar für sie selbst, das war, was sie nicht geahnt hatte, das, was sie nie gewollt und niemals vorhergesehen hatte geschüttelt wie der mächtige Baum, der heftiger erschüttert wird als der schwache Baum nachdem letztlich alle Gänge und Adern geplatzt waren dann ${ }^{4}$

Dieser Romananfang beginnt im inneren Monolog, der etwas von der Bewunderung der Autorin für James Joyce spüren lässt, wie ein Auftakt zu einer Verführungsszene, bei der die Frau ihre Waffen auswählt, mit der sie den Mann verführen kann. Doch ist sogleich die Präsenz der Stimme und Logik von Odysseus da, der ihr bereits seine Meinung zu ihrem Geschmack gesagt hatte, eine Meinung, die keineswegs positiv war und sich ihr eingeprägt hatte. Wir haben es mit einem inneren Monolog in der dritten Person zu tun, eine experimentelle Form, die jenseits der Beziehung zu James Joyces Ulysses die weibliche Stimme ins Zentrum

4 Lispector, Clarice: Uma aprendizagem, S. $19 \mathrm{f}$. 
rückt, die innere Stimme also zu einer weiblichen macht, von der die männliche Stimme, bisweilen auch in direkter Rede, abgesetzt wird. Sofort kommt die Frage der Identität auf, lässt die Stimme von Odysseus doch vermuten, dass die weibliche Figur von einem Identitätsproblem heimgesucht wird und nicht ,ich“ zu sich sagen kann. Parallel zum intertextuellen Verweis auf Ulysses wird im Namen Loreley ironisch und humorvoll zugleich die in Odysseus präsente Schifffahrtsmetaphorik wieder aufgenommen und gespiegelt. Der antike griechische Held trifft zusammen mit seinem irischen Double auf eine Figur der deutschen Romantik, die durch ein Beben in der Erde oder ihrem Uterus erschüttert wird. Homer und James Joyce, aber auch Clemens Brentano sind als Paten zu dieser literarischen Geburt nach Brasilien eingeladen.

Ein weiteres Problem, das in dieser Eingangspassage angesprochen ist, betrifft die Frage des Schmerzes. Denn ein Leben ohne Schmerz, das sie als seine Lehre vermutet, wird konterkariert durch den Schmerz, der Lóris Leib unvermittelt trifft. Gegen den rationalen, aber zugleich patriarchalischen Diskurs des Mannes erhebt sich so, einem Beben gleich, das aus der Tiefe kommt, der des weiblichen Körper-Leibes, der nicht nur von der Stelle des Herzens aus, sondern mehr noch von jener des Uterus geführt wird, also des Weiblichen schlechthin. Der weibliche Leib ist die Bühne für jenes Erleben von Schmerz und Lust, das auf der Ebene eines „Being a Body“ in diesem Buch der Lüste experimentell in Szene gesetzt wird.

Bereits im Incipit kommt es zu einer Erschütterung, von der nicht feststeht, ob sie ausgelöst wurde von jenem Diskurs über das Ich, von dem in der „Nota“ bereits die Rede war. Doch ist diese Kontiguität, die Ihab Hassan kurzerhand zu den Indizien postmodernen Schreibens rechnete, durchaus ein Zeichen für eine möglicherweise ambivalente Beziehung zwischen beiden Ich-Konfigurationen. Was Clarice Lispector an dieser frühen Stelle ihres Experimentaltextes einführte, ist die Sprache des Körpers, mehr noch, die des weiblichen Körpers, welche in den Diskursen der sechziger und siebziger Jahre unter dem Druck neuer feministischer Theoriebildungen in der Tat von größter Bedeutung wurde. Dies erfolgte übrigens nicht allein in einer von Frauen verfassten Literatur: Auch bei Roland Barthes finden wir gerade seit Ausgang der sechziger und dann vor allem in den frühen siebziger Jahren nicht nur die Thematik von Begehren und dann Lust, die an den erotisierenden Text rückgekoppelt ist, sondern die Thematik des Körpers. Denn, so heißt es bei Barthes in Die Lust am Text, „mein Körper hat nicht dieselben Ideen wie ich“5 - ein Satz, den man sehr wohl mit der „Nota“ von Clarice Lispector in einen fruchtbaren Zusammenhang bringen könnte.

5 Barthes, Roland: Die Lust am Text. Frankfurt a.M.: Suhrkamp 1996, S. 26. 
Im weiteren Verlauf des Romans kommt es letztlich zur Erfüllung der Verführungsszene, welche dann freilich keine Verführung mehr sein und darstellen wird. Denn Odysseus hatte seine künftige Geliebte aufgefordert, genau dann bei ihm zu erscheinen, sobald sie für ihn bereits sei: Er werde warten und in der Zwischenzeit mit keiner anderen Frau ein Verhältnis eingehen. So wird sie quasi von einer Naturgewalt, mitten in der Nacht und aus dem Bett heraus, $\mathrm{zu}$ ihm hingezogen, wo es dann zur Vereinigung der Körper-Leiber und mehr noch der Gegensätze kommt, ohne dass letztere aufgelöst würden. Mann und Frau behalten ihre jeweiligen geschlechterspezifischen Rollen bei, doch sie haben Lust aneinander gefunden. Und diese wird in jenem „Buch der Lüste“ dann zu einem zentralen Erleben - und auch an dieser Stelle wäre es ein Leichtes, eine Beziehung zu Theoremen von Roland Barthes herzustellen.

Im Grunde geht es ausschließlich um die Lehrjahre von Lóri, die nicht nur unter der Anleitung des schlauen Odysseus, sondern auch ihrer eigenen Fähigkeit lernt, durch immer neues Erleben neue Erkenntnisse zu produzieren. Sie betrachtet sich dabei nicht nur immer wieder im Spiegel - ein Ritual, das sie sehr ernsthaft vollzieht -, sondern auch in all ihren Handlungen und Bewegungen, so als stünde sie zugleich immer außerhalb ihrer selbst, als betrachtete sie sich von außen. Sie tritt sich immer wieder als einer anderen gegenüber und löst jenes angekündigte Aufklaffen einer Ich-Identität ein, das sich durch den gesamten autofiktionalen Text zieht.

An dieser Stelle erscheint somit eine autobiographische Sichtweise - wenn wir uns auf diese Lesart beschränken - in Form der dritten Person Singular, also keineswegs gattungskonform. Ich verweise ein drittes Mal auf den Experimentaltext Roland Barthes par Roland Barthes, nicht um eine wie auch immer geartete ,Beeinflussung، von Clarice Lispector durch den französischen Zeichentheoretiker und Schriftsteller zu behaupten, sondern um Parallelen aufzuzeigen, die sich zwischen den Texten eines selben Zeitraums ergaben. In diesem auf das weibliche Selbst gerichteten Schreiben erscheint der weibliche Körper-Leib in seinen verschiedensten Inszenierungen immer wieder als jene Fläche und Oberfläche, in welche die verschiedensten kulturellen und a-kulturellen Zeichen eingeschrieben werden können:

- ob sie Klunker anlegen würde? zögerte sie, denn sie wollte ganz fragile und schlichte Ohren, etwas auf bescheidene Weise Nacktes, zögerte noch mehr: ein noch größerer Reichtum wäre es, mit den Haaren die Reh-Ohren zu verstecken und sie verschwiegen zu machen, aber sie konnte nicht widerstehen: sie deckte sie auf und steckte ihre Haare hinter die ungleichmäßigen und blassen Ohren: ägyptische Königin? nein, ganz wie die biblischen Frauen geschmückt, und es gab in ihren bemalten Augen auch etwas, was mit Melancholie sagte: entziffre mich, meine Liebe, oder ich fühle mich gezwungen, dich zu fressen, und 
jetzt bereit, angezogen, so sehr hübsch, wie zu sein es ihr gelang, kamen von neuem der Zweifel, ob sie zum Date mit Odysseus gehen solle oder nicht - bereit, mit hängenden Armen, nachdenklich, würde sie zum Date gehen oder nicht? ${ }^{6}$

Diese Passage ist in vielerlei Hinsicht aufschlussreich: Sie bemerken ohne weiteres, wie ständige (kulturelle) Umkodierungen immer wieder andere, weitere Identitäten aufscheinen lassen, wobei dieses Spiel für das Ich selbst nicht ungefährlich ist. Gegen Ende des Romans wird Odysseus selbst diese gegebene Gefährlichkeit aufdecken und zu ihr just jene Worte sagen, die sie sich als Sphinx selbst im Spiegel gesagt hatte: Entziffere mich, oder ich muss dich fressen. Das Entziffern der Phänomene, die Semiotik der Liebe und des Begehrens, ablesbar am Körperlichen, ist also in gewisser Weise eine überlebensnotwendige Technik für beide Liebespartner. Dabei steht ohne Zweifel der Körper und weniger der Leib im Mittelpunkt, also jener Körper, den ich als Objekt behandeln kann, bearbeiten und bemalen, bestimmte Teile herausstellen oder lieber verstecken kann, um bestimmten (Selbst-) Bildern zu entsprechen. Das Ich macht den Körper zu seinem eigenen Objekt, das als Gegenstand der Liebe herausgeputzt wird. Mit Schmerzen und Lust des eigenen Leibes hat dies noch nichts zu tun.

Uns fehlt leider die Zeit, an Clarice Lispectors überaus einfach konzipierten, zunächst an simplen Grundmustern entwickelten, aber höchst differenziert alles miteinander verwebenden und komplex geschriebenen Text weiterzuarbeiten. Natürlich hat dieser romanhafte Text Ende der sechziger Jahre innerhalb der vorherrschenden Romantraditionen in Brasilien einen Skandal ausgelöst, da er mit vorgängigen Klischees und Stereotypen insbesondere von Weiblichkeit spielt, sie aber zugleich umspielt und hinterfragt, ohne sie doch gänzlich aufzulösen. Es sind, wie wir gerade in dieser Passage sahen, unmerkliche Verschiebungen, kleinste Bewegungen, welche plötzlich neue Horizonte aufscheinen lassen, eine Situation völlig verändern können, ohne dass man doch genau sagen könnte, was diese Veränderung wirklich ausgelöst hat.

Das unsichere, alle durchgängig rationale Logik in Frage stellende Wesen ist die Frau selbst, das weibliche Ich, wobei man im Verlauf des Romans immer deutlicher merkt, dass es schlicht eine andere Logik ist, die sich der männlichen Zeichendeutung und Lehre entgegenstellt, ohne ihr doch völlig zu entgleiten. Dabei ist es, so scheint mir, keine ,eigene', ,weibliche', einheitliche Logik, sondern ein offenes Spiel, eine bewegliche Konfiguration von Logiken, welche sich einer klar eingrenzbaren männlichen Logik zu erkennen und zu verbergen gibt. Wird nicht auch die männliche Logik in die weibliche miteinbezogen, dechiffriert und in

6 Lispector, Clarice: Uma aprendizagem, S. 24. 
Handlungsmuster umgesetzt? Auf diese Weise wird die Diskussion der Geschlechterdifferenz überaus variantenreich ausgeführt: Das Weibliche erscheint schlicht als viellogisch.

Doch ließen sich auch einige Kritikpunkte formulieren. Denn zugleich wird mancher männliche Leser den Eindruck nicht los, dass trotz aller Bewegung und Mobilität die Geschlechterstandorte doch letztlich essentialistisch definiert sind, dass ihnen also ein unhintergehbares ,So-Sein“ eignet. Gleichzeitig wird von der literarischen Formgebung her eine fundamentale Ambivalenz in den literarischen Text gebracht, wo diese Philosopheme immer wieder unterlaufen und auf vorangehende Klischees bezogen werden, die zuvor bereits demontiert wurden.

Auf diese kunstvolle Weise enthält der Text schon von seiner experimentellen literarischen Form her ungeheuer viele Unbestimmtheits- und Leerstellen, was viele Feministinnen der sechziger und siebziger Jahre nicht zufriedenstellen konnte, richtet dieser autofiktionale Roman doch keinerlei Kampfansage an den patriarchalischen männlichen Diskurs in einem offenen, militanten Sinne. Der männliche Diskurs zielt in Gestalt von Odysseus vermittels des männlichen Blicks ja nicht weniger als der weibliche auf den weiblichen Körper, der zentral gestellt wird. Allerdings sind die Formen der Distanzierung - also der Ironie, des Pastiche und der Parodie - so kunstvoll in diesen Text eingewoben, dass es schwerfällt, ihn in einen Thesenroman zurückzuübersetzen. Denn dafür taugt Clarices starker autofiktionaler Roman über komplexe Selbstfindungsprozesse nicht!

Die unhintergehbare Körperlichkeit und Leiblichkeit, die in das Livro dos prazeres offenkundig wesentlich stärker für die Frau und kaum für den Mann gilt, ist eines der zentralen Elemente dieses Textes. Die eigene weibliche Identitätskonstruktion wird damit an den Körper-Leib zurückgebunden und nicht in einem vom Körper distanzierten Diskurs gesucht. Zugleich ist dieser Körper als Objekt aber die Vielförmigkeit, die Vielgestaltigkeit par excellence. Denn er funktioniert in vielerlei Hinsicht wie die plane Oberfläche einer weißen Leinwand, bildet die Projektionsfläche für eigene Wünsche und jene des eigenen Begehrens zum Verführen. Er ist Fläche für Projektionen des männlichen Blickes, insoweit sich Odysseus, wie er später einräumt, schon beim ersten Treffen auf der Straße dachte, „dass du gut in einem Bett sein würdest“. Und er ist über diese erotische Vergegenständlichung als Lustobjekt hinaus die Fläche ständiger kultureller Kodierungen, welche Lóri zu einem Ich und zugleich zur Frau machen, die für alle Frauen steht und doch wiederum nur für sich selbst.

So wird diese brasilianische Loreley, zu deren Füßen übrigens die Schiffer nicht im Rhein, sondern in einem Meer versinken, zum Zeichen eines nach Amerika verpflanzten weiblichen Mythos des Ewig-Weiblichen, aber nicht in der Form des Ausgleichenden und nach Harmonie Strebenden, sondern in Form einer Trägerin essentieller Andersheit, unhintergehbarer Alterität, die an den weibli- 
chen Körper-Leib zurückgebunden ist. Die transatlantischen ,Verpflanzungen“, auf die ich an späterer Stelle in unserer Vorlesung noch eingehen werde, bleiben auf diese Weise in starkem Maße eingeschrieben und stehen der Entzifferung offen. So sagt der Text seinem Spiegel, also uns Leserinnen und Lesern: Entziffre mich oder ich fresse Dich!

Lassen Sie uns nun zu guter Letzt noch auf eine andere Autorin zurückkommen, die wie kaum eine andere dazu befähigt ist, unserer Vorlesung noch einen wichtigen Aspekt autobiographischen und autofiktionalen Schreibens hinzuzufügen! Wir haben den zweiten Teil mit Jorge Luis Borges und seinen Fiktionen aus den dreißiger Jahren abgeschlossen; und ebenfalls schon in den dreißiger Jahren veröffentlichte Nathalie Sarraute ihr erstes, epochemachendes, ihr gesamtes Lebenswerk bereits im Kern enthaltendes Buch mit dem schönen Titel Tropismes. Wie Clarice Lispector war sie letztlich eine Immigrantin, eine Fremde wie Julia Kristeva, mit der wir uns gleich beschäftigen werden, eine zurückgezogen lebende Frau, die noch im hohen Alter mit klarer Stimme ihr fünfzehntes Buch schrieb und vorlas, ein Buch, das sie unter dem Titel Enfance der eigenen Kindheit widmete. Auch in diesem Falle handelt es sich nicht einfach um eine Autobiographie, sondern - um die häufig auch von Nathalie Sarraute gewählte oder bestätigte Bezeichnung zu wählen - um eine „falsche Autobiographie“. Beschäftigen wir uns ganz kurz mit der ,richtigen' Autorin!

Nathalie Sarraute wurde am 18. Juli 1900 im russischen Iwanowo geboren und starb am 19. Oktober 1999 in Paris. Ihr jüdischer Vater hatte an der Genfer Universität in den Naturwissenschaften promoviert und leitete eine Farbstoff-Fabrik in Ivanovo, wo Natalja ihre ersten beiden Lebensjahre verbrachte. Ihre Mutter war Schriftstellerin und arbeitete für eine von Korolenko herausgegebene Zeitschrift. Bald jedoch ließen sich die Eltern scheiden und Nathalie lebte abwechselnd mit der Mutter sowie deren neuem Lebenspartner in der Schweiz oder in Paris, wodurch sie früh Französisch lernte, und mit dem Vater in Ivanovo, zeitweise aber auch in St. Petersburg. Als der Vater aus politischen Gründen das zaristische Russland verließ und nach Frankreich übersiedelte, lebte sie mit ihm in einem gutbürgerlichen, kultivierten Milieu in Paris, wo sie auch ihre von Lektüren geprägte Schulzeit am Lycée Fénelon absolvierte. Die Wiederverheiratung ihres liebevollen Vaters ließ Nathalie die Schwierigkeiten eines Lebens zwischen zwei affektiven Polen und zwei Sprachen mit ganzer Wucht erfahren.

Nach dem Abitur studierte sie an der Sorbonne Anglistik, 1920 folgte ein Studienjahr in Oxford, von 1921 bis 1922 studierte sie Soziologie in Berlin, woran sie ein Jurastudium in Paris anschloss, wo sie 1923 Raymond Sarraute begegnete, den sie zwei Jahre später, nach Abschluss ihres Studiums, heiratete und mit dem sie drei Töchter hatte. Neben gelegentlichen Anwaltstätigkeiten richtete sich ihre eigentliche Leidenschaft spätestens ab 1932 auf die Literatur: Erste Kurztexte 


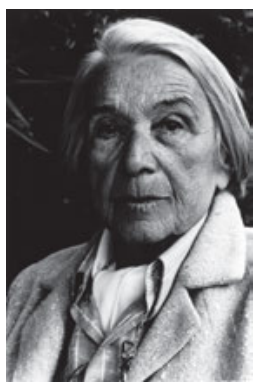

Abb. 111: Nathalie Sarraute (Iwanowo-Wosnessensk, Russland, 1900 Paris, 1999).

der späteren Tropismen wurden verfasst. Doch erst 1939 fand sich für diese von psychologischem Feingespür geprägten Texte ein Verlag, wobei der Band im historischen Kontext des ausbrechenden Zweiten Weltkriegs zunächst unbeachtet blieb. Nach dem Einmarsch der Nazi-Truppen musste die Tochter aus jüdischem Hause aus Paris fliehen und tauchte unter wechselnden Pseudonymen in kleinen Dörfern um Paris unter, wo sie mit viel Glück überlebte.

Selbst im Untergrund schrieb sie, fand allerdings für ihre Texte auch nach dem Krieg nur schwer einen Verlag; doch blieb sie ihrem Schreiben treu und veröffentlichte 1956 unter dem Titel L'Ere du Soupçon einen Band mit Essays, die wie ein Manifest des entstehenden Nouveau Roman gelesen wurden. Es folgten verschiedene Romane, die wie Planétarium von 1959 oder Les fruits d'or von 1963 (erstmals mit einem literarischen Preis ausgezeichnet) ihren literarischen Ruf festigten. Nathalie Sarraute, die sich zuvor ganz der Kindererziehung gewidmet hatte und den Pariser Literaturbetrieb bewusst mied - literarische Freundschaften verbanden sie nur mit Jean-Paul Sartre und Simone de Beauvoir -, unternahm ab Mitte der fünfziger Jahre Reisen in die Sowjetunion, nach Schottland, Italien, Griechenland, Marokko, Spanien, Sizilien, Jugoslawien, Portugal oder Sardinien. Ab 1959 hielt sie Vorträge in der Schweiz, in Belgien, Italien, Schweden, Norwegen, Dänemark, Deutschland, England, in der Sowjetunion, in Kuba und den USA. Spätestens seit Beginn der siebziger Jahre galt die eher zurückgezogen lebende Schriftstellerin als eine der großen französischen Autorinnen der zweiten Hälfte des 20. Jahrhunderts.

Sarrautes Tropismes waren ihrer Zeit weit voraus und nahmen Elemente, Schreibformen und Theoreme des späteren Nouveau Roman zu einem sehr frühen Zeitpunkt vorweg. Wir könnten aus heutiger Sicht und mit dem Vokabular unserer Epoche diese feingliedrigen literarischen Experimente seit den ausgehenden dreißiger Jahren als den Versuch charakterisieren, traditionelle Erzählformen des Romans, herkömmliche Konstruktionsmuster literarischer Figuren und Protagonisten sowie überkommene und vorherrschende Lektüreweisen und Techniken zu dekonstruieren sowie die Autorfunktion als Subjektivität deutlich zu hinterfragen. Das Spannende dabei ist, dass dies von einer anderen theoretischen und 
philosophischen Warte aus erfolgte, als dies später im Zeichen von Tel Quel und des Derrida'schen Dekonstruktivismus vollzogen wurde.

In L'Ere du soupçon war von Nathalie Sarraute das Element des fundamentalen Zweifels, des alles subvertierenden Verdachts stark gemacht worden gegenüber allem Herkömmlichen und Überkommenen. Dabei wurden als Ergebnis gerade die unscheinbaren Bewegungen ohne Tiefe, jene kommunikativen Wortbildungsübungen, die man gemeinhin als Konversation bezeichnet, also die plane Oberfläche einer alltäglichen Kommunikation, in den Vordergrund gerückt und einer eingehenden Analyse und Zersetzung unterzogen. Diese Ebene hatte vor der französischen Schriftstellerin noch niemand ins Zentrum eigener literarischer Arbeit zu stellen gewagt, auch wenn gerade die Konversation - denken wir dabei nur an die literarischen Arbeiten Marcel Prousts - bereits des Öfteren einer literarischen Zersetzungskraft ausgesetzt worden war. Niemand jedoch hatte die Minimalbewegungen in scheinbar alltäglichen Gesprächen mit einer solchen Schärfe analysiert wie jene Autorin, die von Kindsbeinen an in den kurzen Kommunikationsfetzen ihrer Eltern die Tragödien erahnt hatte, welche sie selbst auch betreffen sollten. Die Kindheit spielte eine wichtige Rolle in der persönlichen Bildungsgeschichte der Nathalie Sarraute.

Immer radikaler verstand es die in Russland geborene französische Schriftstellerin, den Roman samt Figuren in seine einzelnen Bestandteile zu demontieren und auf eine Art und Weise erneut zusammenzubauen, dass weder die Problematik von Subjektivität noch jene des Kontinuierlichen und Psychologischen wieder eine bedeutende Rolle einnehmen konnten. Wir werden uns mit der Problematik des Kontinuierlichen noch intensiv auseinandersetzen und fragen, welche Formen und epistemischen Metaphern an die Stelle von Kontinuitäten treten können. Allein schon die Schreibweise Nathalie Sarrautes war eine Absage an jede Art dieses Kontinuierlichen im Text.

Zweifellos reicht der von Jean-Paul Sartre mit Blick auf Sarrautes Roman Portrait d'un inconnu wohlwollend geprägte Begriff des „Anti-Roman“ nicht aus, um den experimentellen Charakter des Sarraute'schen Romanprojekts wiederzugeben. Denn der Roman war in seiner langen Geschichte im Abendland schon immer und grundsätzlich ein „Anti-Roman“, und dies seit seiner Schöpfung im modernen Sinne durch Miguel de Cervantes, der in seinem Don Quijote unter anderem den mittelalterlichen Ritterroman liebevoll verspottete. Das Erstaunliche ist darin zu sehen, dass sich die Romanexperimente Nathalie Sarrautes tatsächlich seit Ausgang des 20. Jahrhunderts mit jenen Entwicklungen in Verbindung bringen lassen, welche sich seit den dreißiger Jahren und verstärkt seit den fünfziger und sechziger Jahren um eine völlige Neuschöpfung des literarischen Schreibens und der Weltsichten im Sinne einer Dezentrierung der herkömmlichen Logik(en) verstehen lassen. 
An eben dieser Stelle berühren wir den Punkt, an dem sich Nathalie Sarraute mit Clarice Lispector trifft: Im Aufzeigen einer anderen und zugleich ,weiteren“ eigenen Logik, des Fremden im Eigenen. Dies ohne dass es bei Sarraute - anders als bei Lispector - der Ansatzpunkt eines spezifisch weiblichen Schreibens wäre, der für die Entwicklung einer experimentierfreudigen viellogischen Schreibweise von so grundlegender Bedeutung wäre wie dies bei der brasilianischen, aber ebenfalls aus einer jüdischen Familie aus Osteuropa stammenden Autorin der Fall war. Nathalie Sarrautes Schreiben ist wie das der Brasilianerin letztlich nicht nur Auflösung, sondern auch Fortsetzung des Romans mit anderen Mitteln. Insofern erfüllen beide Autorinnen die der Gattung des Romans eingeschriebene Tradition des „Anti-Romans“ im Sartre’schen Sinne, gehen in ihren Schreibweisen und in ihren Ansprüchen aber weit über diese unverbindliche Klassifizierung hinaus, indem sie an den autobiographischen Oberflächen des Schreibens experimentieren.

Es mag auf den ersten Blick erstaunlich wirken, dass sich auch Nathalie Sarraute - wie andere Autorinnen und Autoren im Zeichen der Postmoderne, zu der man sie im allgemeinen nicht hinzurechnet - ebenfalls nicht nur dem metaliterarischen Diskurs, sondern auch dem auf das eigene Selbst bezogenen in der Spielart der Autobiographie zuwandte. Doch aus der in unserer Vorlesung entwickelten Sichtweise der Literaturen im Zeichen der Postmoderne ist diese Wendung gerade unter der Maßgabe durchaus verständlich, dass wir die Postmoderne nur als transatlantisches und nicht ursprünglich von den USA, sondern ganz wesentlich durch die Literaturen Lateinamerikas geprägtes Phänomen verstehen müssen. Damit soll keineswegs eine wie auch immer geartete intertextuelle Beziehung zwischen Clarice Lispector und Nathalie Sarraute hergestellt, wohl aber eine gemeinsame literarästhetische Ausrichtung behauptet werden, welche einen wichtigen Beitrag von Seiten weiblicher Schriftstellerinnen zu experimentellen - und noch nicht massenkulturell kommerzialisierten - Entwicklungslinien weltweit beobachtbarer Schreibformen im Zeichen der Postmoderne leistete. Zur Erhärtung dieser These wollen wir uns mit Nathalie Sarrautes autobiographischem Text Enfance beschäftigen!

Sie können diesen wohlgesetzten Text der französischen Schriftstellerin übrigens in einer wunderschönen Aufsprache anhören, die die zum damaligen Zeitpunkt weit über Achtzigjährige mit einer unglaublich frischen Stimme selbst ,komponierte‘: mit einem unnachahmlichen Gefühl für Klang, für das richtige Wort, für die Partitur ihres Sprechens und ihrer Diktion. Ich finde diese Selbstaufsprache einer alt gewordenen, aber jung wirkenden Autorin schlicht hinreißend, zeigt sie uns doch phonotextuell in der ganzen Fülle der Stimme auf, aus welchen Klängen und Rhythmen sich die Schreibweise der zu Unrecht als schwierig zugänglich geltenden Schriftstellerin zusammensetzt. Was in der Schreibweise 
von Clarice Lispector die klangliche Wirkung des Doppelpunktes in seiner Suspension von Logiken ist, das bedeutet für die Schreibweise der französischen Autorin das Spiel mit den drei Pünktchen, welche oft jene semantischen Leerstellen markieren, die den Leserinnen und Lesern gelassen werden. So entsteht ein filigran durchbrochenes autobiographisches Werk, das nicht länger den Kontinuitäten eines autobiographischen Diskurses vertraut, der seit Jean-Jacques Rousseau unter dem Gebot des „tout dire“ stand und autobiographische Texte in einer ständig proliferierenden Fülle aufeinander häufte, hinzufügend und immer wieder neu hinzufügend. Hier indes ist es eine Stimme, die sich aus vielen klanglichen Blickwinkeln allein der Kindheit zuwendet, die im Titel des Bändchens der Grande Dame der französischen Literatur angekündigt wird.

Nathalie Sarrautes „falsche Autobiographie“ Enfance erschien 1983 und bildet so etwas wie den Schlussstein ihres literarischen Gesamtwerks. Es liegt vielleicht in der Natur der Sache, dass die widerwillig dem Nouveau Roman zugerechnete Autorin mit einem autobiographischen Text ein breiteres und weniger spezialisiertes Publikum als mit ihren deutlich experimenteller angelegten Erzähltexten erreichte. Die ersten Seiten von Enfance sind von dieser rezeptionssoziologisch und wohl auch rezeptionsästhetisch veränderten Situation ebenso geprägt wie von der metaliterarischen Erörterung über die Autobiographie oder Formen autobiographischen Schreibens bei einer Schriftstellerin, die sich zeitlebens gegen derartige Gattungsformen gesträubt hatte.

Denn Nathalie Sarraute verwahrte sich stets dagegen, konsistente literarische Figuren zu schaffen oder diese Figuren so auszustatten, dass sie mit einer konkreten außersprachlichen Wirklichkeit, mithin der textexternen Realität, verknüpft und in Beziehung gesetzt werden konnten. Es ist daher spannend, das Incipit dieses Textes genau auf diese Fragestellungen nach konsistenten Figuren zu beziehen und Antworten auf die Beziehung, wenn nicht der Autorin, so doch ihrer Erzählfiguren, zu der von ihr nun verwendeten Literaturgattung zu suchen:

- Nun, Du willst das wirklich machen? „Deine Kindheitserinnerungen aufrufen“ ... Wie diese Worte Dich stören, Du liebst sie nicht. Aber erkenne an, dass dies die einzig passenden Worte sind. Du willst „Deine Erinnerungen aufrufen“ ... da gibt es nichts zu wackeln, das genau ist es.

- Ja, ich kann nichts machen, es ist eine Versuchung für mich, ich weiß nicht warum ...

- Vielleicht ist es ... ist es nicht vielleicht ... man merkt es manchmal nicht ... vielleicht liegt es daran, dass Deine Kräfte nachlassen ...

- Nein, ich glaube nicht ... zumindest fühle ich es nicht ... ${ }^{7}$

7 Sarraute, Nathalie: Enfance. Paris: Gallimard 1983, S. 7. 
Es ist erstaunlich: Von Beginn an treffen wir wie bei Clarice Lispector auf eine Verdoppelung der Ich-Figuren, die in ein Gespräch, einen Dialog vertieft sind. Dieses Auseinanderklaffen hatte schon bei der brasilianischen Autorin eine wechselseitige Spiegelung des Ich ermöglicht; und auch für die französische Schriftstellerin erlaubt dieses Verfahren eine Spiegelung von Spiegelungen, die das Innere von außen und das Äußere von innen zeigen, aber dabei Zwischenpositionen einnehmen, welche nicht eindeutig einem ,Außen' und einem ,Innen` zuzuordnen sind.

Von Beginn an wird der Plan des weiblichen Ich, von ihren Kindheitserinnerungen zu berichten, kritisch in Frage gestellt. Hier lastet die ganze Bürde der theoretischen Erörterungen und Thesen im Umkreis des Nouveau Roman und anderer Ansätze auf einer Erzählerin, die doch dem Subjekt den Kampf angesagt hatte. Doch das eine Ich gesteht dem andern, dass es eine Versuchung sei, der Frau nicht widerstehen könne. Es sei nichts daran zu ändern, der Plan sei gefasst - und in diesem zögerlichen Bericht auf einer Metaebene entsteht bereits der literarische Diskurs einer Erinnerung der Kindheit, die aus dieser zweifachen Perspektivik gespiegelt wird. Metadiskurs und Diskurs durchdringen einander und konfigurieren das eigentliche Schreiben dessen, was die reale Autorin als „falsche Autobiographie“ bezeichnet.

Bei dieser Experimentalform der Autobiographie wird zugleich die Möglichkeit erwogen, dass ein Nachgeben dieser Versuchung mit der Tatsache zu tun haben könnte, dass die Autorin nicht mehr im Vollbesitz ihrer Kräfte wäre, dass sie also nicht länger die Energie aufbringen könnte, dieser Versuchung der Subjektivität zu entsagen. Doch das andere Ich versichert, dass dies keinesfalls der Fall sei, dass sie es zumindest nicht spüren könne, über keine Widerstandskraft mehr zu verfügen. So steht der Entschluss also fest, sich an die Kindheit zu erinnern und diese Kindheitserinnerungen niederzuschreiben.

Nicht ein erzähltes und ein erzählendes Ich begegnen sich hier, wie dies noch in der ,klassischen“ Autobiographie der Moderne der Fall war, als deren Schöpfer Jean-Jacques Rousseau betrachtet werden darf. Vielmehr sind es zwei Ich-Figurationen, die sich immer wieder wechselseitig beleuchten, um daraus ein auf vielen Zeitebenen belebtes Bewegungsbild zu erstellen. Der vermeintliche Essentialismus traditionellen autobiographischen Schreibens wird auf diese Weise von Beginn an unterspült. Dass es sich dabei nicht, wie auf der U4 der französischen Ausgabe zu lesen steht, um „Nathalie Sarraute et son double“ handelt, da natürlich keine der beiden Ich-Positionen mit der textexternen Autorin gleichgesetzt werden kann, versteht sich von selbst.

Kunstvoll gehen mit Enfance bei Nathalie Sarraute ein letztes Mal Metaliterarizität und Autoreflexivität in Form des autobiographischen weiblichen Schreibens eine fruchtbare Verbindung ein, die eine in einzelne Szenen fragmentierte Kindheit auf magische Weise dem Lesepublikum nahezubringen versucht. Gewiss 
sind die eigentlichen Handlungsmomente sehr gering, wird doch die Kindheit in ihrer Totalität und Fülle bewusst nicht erfasst. Aber es sind vielleicht gerade die „Tropismen“, diese unmerklichen und oft doppelt gespiegelten Bewegungen, welche in Form autobiographischer Kindheitsmuster jenes Fremde im Eigenen herausarbeiten, das zum Eigenen geworden ist und gleichwohl nicht aufhört, immer noch fremd zu bleiben.

Dies ist gewiss nicht der Weg psychologischer Tiefe und Aufarbeitung psychoanalytisch ergründbarer Kausalzusammenhänge, welche in die Tiefenschichten von Es und Ich vordringen, sondern die narrative Entwicklung eines diskontinuierlichen Verhältnisses zum eigenen Ich, das sich in der Kontinuität des Fragmentierten seiner Eigenständigkeit bewusst zu werden versucht. Die Grunderfahrung dieses Prozesses ,falschen' autobiographischen Schreibens ist die Erfahrung und wohl mehr noch das Erleben des Fremden im Eigenen und sogar das Fremdwerden des Eigenen, das aber immer auch das Annehmen des Fremden im Eigenen darstellt.

Das Selbst-Erleben einer kontinuierlichen Tiefenpsychologie war der Inbegriff des modernen Menschenbildes, in welchem seit Sigmund Freud stets der Oberfläche des Manifesten das eigentliche ,Wahre“ in der Tiefenschicht entspricht. Dagegen könnten wir in den Autobiographien der achtziger und neunziger Jahre ein Menschenbild sich entwickeln sehen, das nicht minder vielgestaltig ist, ohne doch das Diskontinuierliche stets an ein Kontinuierliches, das Hybride und Heterogene stets an ein Homogenes zurückbinden zu müssen.

Die ,Rückkehr` zum autobiographischen oder autofiktionalen Schreiben ist in diesem Sinne nicht die Rückkehr zum Subjekt, zu dessen Größe und Großartigkeit, sondern dessen diskontinuierliche Fremderfahrung als diskontinuierliches Eigenes. Das Sprechen fremder Sätze macht deutlich, wie sehr sie zu einem eigenen Sprechen, zum Eigenen geworden sind, ohne doch im Spiel der Spiegelungen ihre ferne Fremdheit verloren zu haben: Wir wohnen einer Einverleibung bei, die in den eigenen (textuellen) Leib das einfügt, was als Fremdes zum Eigenen wird und als ,richtige' Autobiographie zur falschen gerät. Einverleibt in den Klangkörper der Autorin und ihrer Stimme(n), wird nochmals in den letzten Sätzen des Textes das gespiegelt, worum es in diesem wahren AutobiographieExperiment geht:

Sei ganz beruhigt, ich bin fertig, ich ziehe Dich nicht mehr weiter mit hinein ...

- Warum jetzt so plötzlich, wo Du doch keine Angst davor hattest, bis hierher zu kommen?

- Ich weiß nicht genau ... ich habe keine Lust mehr ... ich würde gerne woandershin gehen ... Vielleicht ist es, weil es mir scheint, dass für mich hier die Kindheit aufhört ... Wenn ich mir anschaue, was sich mir jetzt bietet, dann sehe ich so etwas wie einen enormen Raum, der sehr voll ist und gut ausgeleuchtet ... 
Ich könnte mich nicht zwingen, einige Momente, einige Bewegungen auftauchen zu lassen, die mir noch als intakt erscheinen, stark genug, um sich von dieser schützenden Schicht zu lösen, welche sie bewahrt, von diesen weißlichen, weichlichen, wattebauschartig Weiten, das sich auflöst, das verschwindet mit der Kindheit ... ${ }^{8}$

Die Kindheit ist zu Ende, und das Ich hat zum Schreiben keine Lust mehr. Die große Versuchung, Kindheitserinnerungen zu erzählen und zu präsentieren, ist vorbei. Das Ich quittiert den Dienst und will woanders hin. Ein langes Leben, ein langes Schreiben, klingt mit einem Leben-Schreiben aus, das im hohen Alter zur Kindheit zurückführt. Der Kreis des Erzählens hat sich geschlossen, ohne eine Totalität, ein gesamtes Leben in seiner Kontinuität, umschlossen zu haben.

8 Sarraute, Nathalie: Enfance, S. 277. 